\title{
SEMIGROUPS CORRESPONDING TO ALGEBROID BRANCHES IN THE PLANE
}

\section{H. BRESINSKY}

AbSTRACT. The symmetric semigroups of nonnegative integers and their generators, corresponding to algebroid branches of the plane, are determined.

Let $\alpha$ be an algebroid branch of a plane curve with coefficients in an algebraically closed field with characteristic 0 . Although the semigroup $S(\alpha)$ of $\alpha$ is symmetric, not all symmetric semigroups correspond to branches [1]. Subsequently the nonredundant generators of $S(\alpha)$ are found from which follow necessary and sufficient conditions for a nonredundant set of positive integers to generate the semigroup of a branch $\alpha$. A method for obtaining the generators from the power series is given.

The definitions for infinitely near points $P_{j}$, multiplicity sequence, proximity structure, satellite cluster and multiplicity matrix are given in [3]. By the restriction of a point $P_{j}$ we mean the number of points $P_{j}$ is proximate to, and the leading points are the points which have successors with increased restriction. Defining the order of a divisor $D$ on $\alpha, o(D, \alpha)$, in the usual way, let $v\left(P_{j}, \alpha\right)=\min (o(D, \alpha))$, where $\alpha$ passes thru $P_{j}$ and $D$ is a divisor of $P_{j}$.

LEMMA 1. If $\alpha$ has satellite clusters $S_{1}, \cdots, S_{n}$ and $\alpha^{*}$ is obtained from $\alpha$ by deleting $S_{n}$, then $v\left(P_{j}, \alpha\right)=c v\left(P_{j}, \alpha^{*}\right), 0 \leqq j \leqq l, P_{l}$ the last satellite point of $\alpha^{*}$.

Proof. Let $M(\alpha)=\left(m_{i j}\right), 0 \leqq j \leqq t$, be the upper triangular multiplicity matrix of $\alpha$. Then $v\left(P_{j}, \alpha\right)=\sum_{i=0}^{t} m_{i j} m_{i t}, j \leqq t$ [2]. Since $M\left(\alpha^{*}\right)=$ $\left(m_{i j}^{*}\right)$ consists of the first $l$ rows and columns of $M(\alpha)$, by using the proximity structure, for $j \leqq l$,

$$
\sum_{i=0}^{t} m_{i j} m_{i t}=\sum_{i=0}^{l} m_{i j}^{*}\left(m_{l t} m_{i l}^{*}\right)=m_{l t} v\left(P_{j}, \alpha^{*}\right) .
$$

LEMma 2. If $L_{j}$ are the leading points, $0 \leqq j \leqq n$, then

$$
\text { g.c.d. }\left(v\left(L_{0}, \alpha\right), \cdots, v\left(L_{n}, \alpha\right)\right)=d=1 \text {. }
$$

Received by the editors January 12, 1971.

AMS 1970 subject classifications. Primary 14B99, 14N99.

Key words and phrases. Algebroid branch of a plane curve, semigroup of a branch, infinitely near points, multiplicity sequence, multiplicity matrix, proximity structure, satellite cluster, free point, restriction, leading point.

(c) American Mathematical Society 1972 
Proof. If $\alpha$ has a single satellite cluster, then g.c.d. $\left(v\left(L_{0}, \alpha\right), v\left(L_{1}, \alpha\right)\right)=$ $m_{t t}=1$. Let $\alpha$ have $n$ clusters. Assuming the truth for $\alpha^{*}$, defined in Lemma 1 , implies $d \mid m_{l t}$. Let $L_{n+1}=P_{r+1}$. Since $v\left(P_{r+1}, \alpha\right)=\sum_{i=0}^{r} m_{i r}$ $\cdot m_{i t}+m_{r+1, t}$ and since $m_{l t}\left|m_{i t}, 1 \leqq i \leqq r, d\right| m_{r+1, t} \Rightarrow d \mid$ g.c.d. $\left(m_{l t}, m_{r+1, t}\right)=1$.

COROLlaRY 1. Let $\alpha_{j}$ be the branch whose only satellite cluster is $S_{j}$, $1 \leqq j \leqq n$, with multiplicity $\lambda_{j}$ at the origin and let $\lambda_{n+1}=1$. Then $v\left(L_{0}, \alpha\right)=$ $\prod_{i=1}^{n+1} \lambda_{i}$ and g.c.d. $\left(v\left(L_{0}, \alpha\right), \cdots, v\left(L_{j}, \alpha\right)\right)=\prod_{i=j+1}^{n+1} \lambda_{i}$.

Lemma 3. Let $\alpha$ have a single satellite cluster. Let $L_{1}=P_{r+1}$. Then $m_{r t} m_{r+1, t}=\sum_{i=r+1}^{t}\left(m_{i t}\right)^{2}$.

Proof.

$$
\begin{aligned}
m_{r t} & =q \cdot m_{r+1, t}+m_{r+1+\alpha, t} \\
& \Rightarrow m_{r t} m_{r+1, t}=\sum_{i=r+1}^{r+q}\left(m_{i t}\right)^{2}+m_{r+1, t} m_{r+1+q, t} .
\end{aligned}
$$

Repeating the process with $m_{r+1, t}$ and $m_{r+1+a, t}$ proves Lemma 3.

LEMMA 4. Let $E_{j}$ denote the last satellite point of the jth cluster of a branch $\alpha$ with $n$ clusters. Then $v\left(E_{j}, \alpha\right)=\lambda_{j} v\left(L_{j}, \alpha\right)$.

Proof. Assume $j=n$ and $L_{n}=P_{r+1}$. If $P_{q}$ is proximate to $P_{q-1}$ and $P_{a-k}$, then $m_{a q}=1$ and $m_{s q}=m_{s, q-1}+m_{s, q-k}, s<q$. Therefore the proximity structure and Lemma 3 imply

$$
\begin{aligned}
v\left(E_{n}, \alpha\right) & =\left(m_{r t}-m_{r+t, t}\right) v\left(P_{r}, \alpha\right)+m_{r+1, t} v\left(P_{r+1}, \alpha\right)+\sum_{i=r+2}^{t}\left(m_{i t}\right)^{2} \\
& =\left(m_{r t}-m_{r+1, t}\right)\left(v\left(P_{r}, \alpha\right)+m_{r+1, t}\right)+m_{r+1 . t} v\left(P_{r+1}, \alpha\right) \\
& =\lambda_{n} v\left(L_{n}, \alpha\right) .
\end{aligned}
$$

This and Lemma 1 also prove Lemma 4 , if $j \neq n$.

CoROllaRY 2. If $n \geqq 2$, then $\sum_{j=1}^{n-1}\left(\lambda_{j}-1\right) v\left(L_{j}, \alpha\right)<v\left(L_{n}, \alpha\right)$.

Proof. Induction on $n$.

LEMMA 5. If $\sum_{j=1}^{n} a_{j} v\left(L_{j}, \alpha\right)=\sum_{j=0}^{n} b_{j} v\left(L_{j}, \alpha\right), a_{j}$ and $b_{j}$ nonnegative integers, and if $0 \leqq a_{j}<\lambda_{j}$, then $b_{0}=0$ and $a_{j}=b_{j}, 1 \leqq j \leqq n$.

Proof. For one satellite cluster the statement follows from g.c.d. $\left(v\left(L_{0}, \alpha\right), v\left(L_{1}, \alpha\right)\right)=1$. Assume $\alpha$ has $n \geqq 2$ clusters and assume two different representations

$$
\sum_{j=1}^{n-1} a_{j} v\left(L_{j}, \alpha\right)+a_{n} v\left(L_{n}, \alpha\right)=\sum_{j=0}^{n-1} b_{j} v\left(L_{j}, \alpha\right)+b_{n} v\left(L_{n}, \alpha\right) .
$$


By Corollary 2, $b_{n} \leqq a_{n} \Rightarrow a_{n}=b_{n}$, since $\lambda_{n} \mid a_{n}-b_{n} \Rightarrow \sum_{j=1}^{n-1} a_{j}\left(v\left(L_{j}, \alpha\right) / \lambda_{n}\right)=$ $\sum_{j=0}^{n-1} b_{j}\left(v\left(L_{j}, \alpha\right) / \lambda_{n}\right)$, a contradiction if the truth is assumed for $n-1$ clusters.

THEOREM 1. $S(\alpha)$ is generated nonredundantly by $v\left(L_{j}, \alpha\right), 0 \leqq j \leqq n$.

Proof. By Lemma 5, $\sum_{j=1}^{n} a_{j} v\left(L_{j}, \alpha\right), 0 \leqq a_{j}<\lambda_{j}$, are distinct first elements of $S(\alpha)$ in the congruence classes $\bmod \left(v\left(L_{0}, \alpha\right)\right), \prod_{j=1}^{n} \lambda_{j}=$ $v\left(L_{0}, \alpha\right)$ in number.

Let $S=\left\{a_{0}<a_{1}<\cdots<a_{n}\right\}$ be a set of nonredundant integers, $n \geqq 1$. Let $\lambda_{n+1}=$ g.c.d. $\left(a_{0}, \cdots, a_{n}\right)=1$ and recursively

$$
\begin{gathered}
\lambda_{j}=\text { g.c.d. }\left(a_{0}\left|\Pi_{j+1}, \cdots, a_{j-1}\right| \Pi_{j+1}\right), \quad 2 \leqq j \leqq n, \\
\Pi_{j+1}=\prod_{i=j+1}^{n+1} \lambda_{i}, \quad 1 \leqq j \leqq n .
\end{gathered}
$$

Let $\lambda_{1}=a_{0} \mid \Pi_{2}$. From Lemmas 1 and 4 then follows

THEOREM 2. $S$ generates a semigroup $S(\alpha)$ iff $\lambda_{j}>1,2 \leqq j \leqq n$, and $\lambda_{j} a_{j}<a_{j+1}, 1 \leqq j \leqq n-1$.

Let $\alpha$ have representative

$$
\begin{aligned}
x_{\alpha}=u^{v_{0}}, \quad y_{\alpha} & =\sum_{h=1}^{g(0)} a_{h, 0} u^{h \cdot v_{0}}+\sum_{h=1}^{g(1)} a_{h, 1} u^{v_{1}+(h-1) d_{1}} \\
& +\cdots+\sum_{h=1}^{g(n-1)} a_{h, n-1} u^{v_{n-1}+(h-1) d_{n-1}}+a_{1, n} u^{v_{n}}+\sum_{h=1}^{\infty} a_{h} u^{v_{n}+h}
\end{aligned}
$$

where $a_{1, j} \neq 0,1 \leqq j \leqq n, 1<d_{j}=$ g.c.d. $\left(v_{0}, \cdots, v_{j}\right), 1 \leqq j \leqq n-1,1=d_{n}=$ g.c.d. $\left(v_{0}, \cdots, v_{n}\right)$.

Then $d_{j}=\prod_{i=j+1}^{n+1} \lambda_{i}, v_{0}=\prod_{i=1}^{n+1} \lambda_{i}, \lambda_{i}$ defined in Corollary $1, v_{1}=$ $v\left(L_{1}, \alpha\right)$ and for $2 \leqq j \leqq n, v_{j}=m(j)+v_{j-1}+k_{j-1} d_{j}$, where $m(j)$ is the multiplicity of $L_{j}$ and $k_{j-1}+1$ is the number of free points following the $(j-1)$ st cluster [3, Chapter XI, §6.1]. Hence $v_{j}=v\left(L_{1}, \alpha\right)+\sum_{i=2}^{j}\left[m(i)+k_{i-1} d_{i}\right]$. By Lemma 4,

$$
\begin{aligned}
& \lambda_{i-1} v\left(L_{i-1}, \alpha\right)+k_{i-1} d_{i}+m(i)=v\left(L_{i}, \alpha\right) \\
& \Rightarrow v_{j}=\sum_{i=1}^{j} v\left(L_{i}, \alpha\right)-\sum_{i=2}^{j} \lambda_{i-1} v\left(L_{i-1}, \alpha\right) \\
& \Rightarrow v\left(L_{j}, \alpha\right)=v_{j}+\sum_{i=2}^{j}\left(\lambda_{i-1}-1\right) v\left(L_{i-1}, \alpha\right),
\end{aligned}
$$

which determines the generators recursively. 


\section{REFERENCES}

1. R. Apéry, Sur les branches superlinéaires des courbes algébriques, C. R. Acad. Sci. Paris 222 (1946), 1198-1200. MR 8, 221.

2. M. A. Hoskin, Zero-dimensional valuation ideals associated with plane curve branches, Proc. London Math. Soc. (3) 6 (1956), 70-99. MR 17, 665.

3. J G. Semple and G. T. Kneebone, Algebraic curves, Oxford Univ. Press, London, 1959. MR 23 \#A2111.

Arizona State University, Tempe, Arizona 85281

UNIVERSITY OF MAINE, ORONo, MAINE 04473 (Current address) 